\title{
Subtypes of pancreatic stellate cells and distant metastasis of pancreatic ductal adenocarcinoma
}

\author{
Yuhui Fan ${ }^{1}$, Marina Lesina ${ }^{1}$, Hana Algül ${ }^{1,2}$ \\ ${ }^{1}$ Department of Gastroenterology, Internal Medicine II, Klinikum rechts der Isar, ${ }^{2}$ Comprehensive Cancer Center MünchenTUM, Technical \\ University of Munich (TUM), Munich, Germany \\ Correspondence to: Hana Algül, Univ.-Prof. Dr. med, MPH. Director Comprehensive Cancer Center Munich at the Klinikum rechts der Isar, Mildred- \\ Scheel-Chair of Tumor Metabolism, Technical University of Munich, Ismaninger Str. 22,81675 Munich, Germany. Email: hana.alguel@mri.tum.de. \\ Provenance and Peer Review: This article was commissioned by the editorial office, Annals of Translational Medicine. The article did not undergo \\ external peer review. \\ Comment on: Wen Z, Liu Q, Wu J, et al. Fibroblast activation protein alpha-positive pancreatic stellate cells promote the migration and invasion of \\ pancreatic cancer by CXCL1-mediated Akt phosphorylation. Ann Transl Med 2019;7:532.
}

Submitted Jan 24, 2020. Accepted for publication Feb 25, 2020.

doi: 10.21037/atm.2020.03.136

View this article at: http://dx.doi.org/10.21037/atm.2020.03.136

Pancreatic ductal adenocarcinoma (PDAC) is now the third most leading cause of cancer-related mortality in the USA (1), and the 5 -year survival rate is mere $9 \%$ (2). Although surgical resection might be potentially curative therapies for some early patients with PDAC, the recurrence rate is quite high, with the median overall survival varies between 24-30 months (1) and the 5-year survival rate of resected patients is only about $20 \%$ (3). In addition to a few cases, monotherapy with immune checkpoint inhibitors, targeted monoclonal antibodies or receptor tyrosine kinase-targeted therapies have been showed ineffective in the clinical treatment for this disease (4).

The poor prognosis of PDAC is mainly due to inefficient diagnosis and tenacious drug resistance. The extracellular matrix (ECM), as the main component of the PDAC stroma, provides biophysical and biochemical cues to regulate malignant cell behavior (5). Abnormal ECM in the tumor microenvironment prompts cancer progression by promoting cellular transformation and metastasis, influences stromal cell behaviors, such as inflammation and angiogenesis, and can intensify the formation of a tumorigenic microenvironment (6). ECM proteins have also been regarded as significant part of the metastatic niche to enable the growth of the metastasis-initiating cells (7). In the normal pancreas, pancreatic stellate cells (PSCs) account for $4 \%$ of the total number of cells, and are mainly located around the acinus and interlobular space of the pancreas (8). PSCs are the major constitutive component of pancreatic cancer stroma. Pancreatic cancer cells (PCCs) release mitogenic and pro-fibrogenic stimulators, which can lead to the activation of PSCs (9). The activation of PSCs and the development of dense stroma are prominent features of PDAC, which illustrates the aggressiveness of PDAC $(10,11)$. Activated PSCs secrete a variety of cytokines that regulate the tumorigenesis, metastasis and chemotherapy resistance of pancreatic cancer (12). The interaction between PSCs and PCCs not only promotes tumor progression and metastasis, but also maintains PSCs activation, and results in a vicious cycle which intensifies PDAC tumorigenesis and drug resistance (13-18).

All in all, the outcomes for PDAC remain dismal and new therapies are urgently needed (1). To date, it is still obscure whether PSCs regulates the progression of PDAC, we tend to believe that figure out the communication between PSCs and PCCs could contribute to develop early detection methods and novel therapeutic options for PDAC.

The pancreatic stromal TGF $\beta$ regulates tumor-related PSCs and accelerates the development of PDAC. Briefly, by secreting TGF $\beta 1$, tumor cells mediate the conversion of fibroblasts into myofibroblasts, which in turns promote the migration, invasion and epithelial-mesenchymal transition (EMT) of tumor cells. Chemokines are a family of proteins with low molecular weight, which can attract leukocytes (such as monocytes and neutrophils) from blood circulation to the infected or damaged site, and they are believed to 
play fundamental roles in various biological processes including inflammation, angiogenesis, immune response and so on. CXC family chemokines is critical responsible for the cellular biological roles mentioned above (19). Among them, CXCL1 and its receptor CXCR2 are highly expressed in PCC lines and pancreatic cancer tissues (20). The specific molecular mechanism leading to PDAC metastasis is still unknown. Some key signaling pathways, for example, PI3K/Akt signaling pathways, substantially contributes to regulate cell proliferation, apoptosis, angiogenesis, immune suppression, invasion, and metastasis (21). High level of Akt expression can induce EMT and enhance the invasion and metastasis ability of squamous cell carcinoma. Additionally, Akt signaling mediates tumor necrosis factor (TNF)-enhanced endothelial cell migration and tumor angiogenesis (22).

In this Journal, Zhang et al. explored the interaction between PSCs and PCCs, and elucidated the relationship between fibroblast activation protein $\alpha$-positive (FAP $\alpha+)$ PSCs and the clinicopathological characteristics of PDAC. What's more, the authors discussed the effects of FAP $\alpha+$ PSCs in PDAC and the underlying mechanism.

By performing tissue microarray analysis, the author found that FAP $\alpha$ was mainly expressed in the PSCs. The higher number of FAP $\alpha+$ PSCs predicts a higher lymph node metastasis and poorer survival. PCCs can release TGF $\beta 1$ and induce PSCs to express FAP $\alpha$. The authors further explored the effects of FAP $\alpha+$ PSCs on the biological behavior of PDAC in vitro and in vivo. Cytokine chips was performed to measure the differential expression of cytokines in FAP $\alpha+$ and FAP $\alpha$-PSCs. In addition, the phosphotyrosine kinase receptor protein chip was used to detect the phosphorylated tyrosine kinase receptors. Finally, immunoprecipitation method was used to detect the interaction between differential cytokines and tyrosine kinase receptors. They found that, compared with FAP $\alpha-$ PSCs, FAP $\alpha+$ PSCs exerted greater potential to promote the migration, invasion and metastasis of PCCs. Additionally, FAP $\alpha+$ PSCs secreted considerable amount of CXCL1, which binds to CXCR2 and activates the tyrosine kinase receptors EphB1 and EphB3 in PCCs, results in phosphorylation of the downstream Akt and finally promotes the migration and invasion of PCCs. What's more, an FAP $\alpha$ inhibitor named talabostat (PT100) could inhibit the effects of FAP $\alpha+$ PSCs on PCCs and PDAC. This is the first study that reveals the interaction between FAP $\alpha+$ PSCs and PCCs, and elucidates their role in PDAC progression.
FAP $\alpha$ is a kind of membrane serine peptidase that belongs to the type II serine protease family, thus FAP $\alpha$ has the proteolytic activity and can mediate the cleavage of some peptides and cytokine receptors (23). Studies have shown that FAP $\alpha$ is selectively expressed in fibroblasts in the malignant stroma (24), and the proteolytic activity endows $\mathrm{FAP} \alpha$ to promote tumor cells growth and metastasis (25).

Notably, the authors have demonstrated that FAP $\alpha+$ PSCs play a significant role in the migration, invasion, and metastasis of PDAC through Akt signaling pathway, which also indicates a new mechanism that FAP $\alpha+$ PSCs interact with PCCs. Collectively, this study has revealed the molecular mechanisms underlying the cell biological functions of FAP $\alpha+$ PSCs and the interaction between FAP $\alpha+$ PSCs with PCCs, which shed light on a therapeutic target for PDAC treatment.

Actually, TGF- $\beta 1$, which was mentioned in this paper, has been considered as one of the most important cytokines and plays a vital role in regulating the invasion and metastasis of the advanced tumors. For example, in the classic TGF $\beta$-T $\beta$ R-Smads pathway, TGF- $\beta$ activates TGF $\beta$ I, and TGF $\beta R$ I phosphorylates downstream R-Smads (Smad2 and Smad3). R-Smads combines with Co-Smad (Smad4) to form a complex and enters the nucleus and which in turn affects transcription and cell movement. Another classic pathway is TGF $\beta-T \beta R-T A B 1 / T A K 1-$ MKK3-p38. In addition to these two pathways, TGF- $\beta$ can also affect the expression of transcription factors such as Snail1/2, Slug, ZEB1/2, and HMGA2 through TRAF6, PI3K/AKT pathways, and promote EMT. What's more, TGF- $\beta$ can promote tumor development and metastasis by affecting the tumor microenvironment. All these indicate that TGF $\beta R$ is a potential tumor treatment target.

The TGF- $\beta 1$ signaling is eliciting increasing attention in cancer therapy. TGF $\beta 1$ primarily binds to the type II receptor (T $\beta \mathrm{R} I \mathrm{I})$, and then the complex recruits type I receptor (T $\beta R$ I or ALK5), and subsequently activates the canonical TGF- $\beta 1$ signaling by phosphorylating the receptor associated Smads. In recent years, several therapeutic approaches have been tried to target the TGF- $\beta 1$ signaling, among which, several TGF- $\beta 1$ inhibitors targeting TGF- $\beta 1$ receptors have reached the clinics. For example, galunisertib (LY2157299) is an ALK5 inhibitor which has been investigated as a single drug or in combination with Gemcitabine. In addition, Vactosertib is a newly developed ALK5 inhibitor which is also investigated in clinical trial (ClinicalTrials.gov Identifier: NCT04258072). Thus, we might develop therapeutic 
approaches according to the results of these clinical trials. Besides, it is also worthy to investigate the effect of FAP $\alpha$ in PSCs by direct FAP $\alpha$ knock in.

That's what we suggest the authors to do in the future research direction of TGF $\beta 1$. In this way, we might develop effective therapeutic target to control distant metastasis of PDAC.

\section{Acknowledgments}

Funding: This study was financially supported by the China Scholarship Council (CSC) (YF, No.: 201908080017).

\section{Footnote}

Conflicts of Interest: All authors have completed the ICMJE uniform disclosure form (available at http://dx.doi. org/10.21037/atm.2020.03.136). YF serves as an unpaid Section Editor of Annals of Translational Medicine from Jan 2020 to Dec 2021. The other authors have no conflicts of interest to declare.

Ethical Statement: The authors are accountable for all aspects of the work in ensuring that questions related to the accuracy or integrity of any part of the work are appropriately investigated and resolved.

Open Access Statement: This is an Open Access article distributed in accordance with the Creative Commons Attribution-NonCommercial-NoDerivs 4.0 International License (CC BY-NC-ND 4.0), which permits the noncommercial replication and distribution of the article with the strict proviso that no changes or edits are made and the original work is properly cited (including links to both the formal publication through the relevant DOI and the license). See: https://creativecommons.org/licenses/by-nc-nd/4.0/.

\section{References}

1. Siegel RL, Miller KD, Jemal A. Cancer statistics, 2018. CA Cancer J Clin 2018;68:7-30.

2. Jemal A, Bray F, Center MM, et al. Global cancer statistics. CA Cancer J Clin 2011;61:69-90.

3. Miller KD, Siegel RL, Lin CC, et al. Cancer treatment and survivorship statistics, 2016. CA Cancer J Clin 2016;66:271-89.

4. Garrido-Laguna I, Hidalgo M. Pancreatic cancer: from state-of-the-art treatments to promising novel therapies.
Nat Rev Clin Oncol 2015;12:319-34.

5. Grippo PJ, Fitchev PS, Bentrem DJ, et al. Concurrent PEDF deficiency and Kras mutation induce invasive pancreatic cancer and adipose-rich stroma in mice. Gut 2012;61:1454-64.

6. Cukierman E, Bassi DE. Physico-mechanical aspects of extracellular matrix influences on tumorigenic behaviors. Semin Cancer Biol 2010;20:139-45.

7. Oskarsson T, Acharyya S, Zhang XH, et al. Breast cancer cells produce tenascin $\mathrm{C}$ as a metastatic niche component to colonize the lungs. Nat Med 2011;17:867-74.

8. Bachem MG, Schneider E, Gross H, et al. Identification, culture, and characterization of pancreatic stellate cells in rats and humans. Gastroenterology 1998;115:421-32.

9. Wen Z, Liu Q, Wu J, et al. Fibroblast activation protein alpha-positive pancreatic stellate cells promote the migration and invasion of pancreatic cancer by CXCL1mediated Akt phosphorylation. Ann Transl Med 2019;7:532.

10. Chu GC, Kimmelman AC, Hezel AF, et al. Stromal biology of pancreatic cancer. J Cell Biochem 2007;101:887-907.

11. Feig C, Gopinathan A, Neesse A, et al. The pancreas cancer microenvironment. Clin Cancer Res 2012;18:4266-76.

12. Hwang RF, Moore T, Arumugam T, et al. Cancerassociated stromal fibroblasts promote pancreatic tumor progression. Cancer Res 2008;68:918-26.

13. Omary MB, Lugea A, Lowe AW, et al. The pancreatic stellate cell: a star on the rise in pancreatic diseases. J Clin Invest 2007;117:50-9.

14. Mahadevan D, Von Hoff DD. Tumor-stroma interactions in pancreatic ductal adenocarcinoma. Mol Cancer Ther 2007;6:1186-97.

15. Erkan M, Adler G, Apte MV, et al. StellaTUM: current consensus and discussion on pancreatic stellate cell research. Gut 2012;61:172-8.

16. Apte MV, Wilson JS, Lugea A, et al. A starring role for stellate cells in the pancreatic cancer microenvironment. Gastroenterology 2013;144:1210-9.

17. Tape CJ, Ling S, Dimitriadi M, et al. Oncogenic KRAS Regulates Tumor Cell Signaling via Stromal Reciprocation. Cell 2016;165:910-20.

18. Ruess DA, Heynen GJ, Ciecielski KJ, et al. Mutant KRASdriven cancers depend on PTPN11/SHP2 phosphatase. Nat Med 2018;24:954-60.

19. Vinader V, Afarinkia K. The emerging role of CXC chemokines and their receptors in cancer. Future Med 
Chem 2012;4:853-67.

20. Kuwada Y, Sasaki T, Morinaka K, et al. Potential involvement of IL-8 and its receptors in the invasiveness of pancreatic cancer cells. Int J Oncol 2003;22:765-71.

21. Fan Y, Du Z, Steib CJ, et al. Effect of SEPT6 on the biological behavior of hepatic stellate cells and liver fibrosis in rats and its mechanism. Lab Invest 2019;99:17-36.

22. Grille SJ, Bellacosa A, Upson J, et al. The protein kinase Akt induces epithelial mesenchymal transition and promotes enhanced motility and invasiveness of squamous cell carcinoma lines. Cancer Res 2003;63:2172-8.

23. Ajami K, Abbott CA, Obradovic M, et al. Structural

Cite this article as: Fan Y, Lesina M, Algül H. Subtypes of pancreatic stellate cells and distant metastasis of pancreatic ductal adenocarcinoma. Ann Transl Med 2020;8(11):671. doi: 10.21037/atm.2020.03.136 requirements for catalysis, expression, and dimerization in the CD26/DPIV gene family. Biochemistry 2003;42:694-701.

24. Scanlan MJ, Raj BK, Calvo B, et al. Molecular cloning of fibroblast activation protein alpha, a member of the serine protease family selectively expressed in stromal fibroblasts of epithelial cancers. Proc Natl Acad Sci U S A 1994;91:5657-61.

25. Goscinski MA, Suo Z, Florenes VA, et al. FAP-alpha and uPA show different expression patterns in premalignant and malignant esophageal lesions. Ultrastruct Pathol 2008;32:89-96. 